\title{
Introduction to the issue in honor of Sidney Blatt
}

\author{
Osmano Oasi, ${ }^{1}$ John Auerbach ${ }^{2,3}$ \\ ${ }^{1}$ Department of Psychology, Catholic University of the Sacred Heart, Milan, Italy; ${ }^{2}$ Psychology Service, Malcom Randall Veterans \\ Affairs Medical Center, Gainesville, FL; ${ }^{3}$ Department of Psychiatry, University of Florida, Gainesville, FL, USA
}

I have asked John Auerbach to join me in presenting this special issue dedicated to Sidney Blatt. The reasons that prompted this are both biographical, considering the meaningful bond between John and the late Sidney, and scientific, which John and I share in paying a tribute to the many contributions of this master in our field. The impulse and currents of research inspired by Blatt are clearly exceptional, as is clear from the sheer variety of topics that feature in this special issue. But I am getting ahead of myself.

John Auerbach's paper (2017) may indeed be taken as an introduction. He presents us with an extraordinary portrait of Sidney Blatt, from both a personal and an intellectual perspective. We are thus given clear insight into his elaborate education and training, as well as into his most significant areas of research. As these areas are dealt with in the other contributions to this issue, we will mention them only briefly: the two-configurations model, the cognitive morphology of mental representation, and the theory of internalization. Likewise, it would be wrong to underestimate the impetus given by Blatt's work to re-

Correspondence: Osmano Oasi, Department of Psychology, Catholic University of the Sacred Heart, Largo A. Gemelli 1, 20123 Milan, Italy.

Tel: +39.02.7234.2688 - Fax: +39.02.7234.2280

E-mail: osmano.oasi@unicatt.it

Citation: Oasi, O., \& Auerbach, J. (2017). Introduction to the issue in honor of Sidney Blatt. Research in Psychotherapy: Psychopathology, Process and Outcome, 20(1), 1-2. doi: 10.4081/ripppo.2017.268

Key words: Sidney Blatt; Research in Psychotherapy: Psychopathology, Process and Outcome; Depression.

Received for publication: 6 March 2017

Accepted for publication: 6 March 2017.

This work is licensed under a Creative Commons Attribution NonCommercial 4.0 License (CC BY-NC 4.0).

(C) Copyright O. Oasi and J. Auerbach, 2017

Licensee PAGEPress, Italy

Research in Psychotherapy:

Psychopathology, Process and Outcome 2017; 20:1-2

doi:10.4081/ripppo.2017.268 search in psychotherapy, particularly in the field of depression.

Lingiardi, McWilliams, and Muzi (2017) document the importance of Blatt's theoretical and clinical contributions in a paper dedicated to the role of his model of personality in the PDM-2. As the authors argue the dialectical interaction between anaclitic and introjective, or relational and self-definitional, dimensions represents a fruitful way of reading both normal and pathological personality development. But there is more. This dialectic sheds light on a way of understanding mental functioning and appraising its strengths and weaknesses, thus proving extremely useful from a clinical standpoint. Noteworthy implications for treatment methods and the psychotherapeutic process are detailed.

Miller, Hilsenroth and Hewitt (2017) present a review of studies on the relationship between therapeutic alliance and perfectionism. Diverse studies have pointed out how this personality trait - which is linked to the self-criticism dimension - interferes in various ways with the psychotherapeutic process and its outcome. The authors stress the significance of studies conducted on this topic by Blatt and colleagues, starting with those in the Treatment of Depression Collaborative Research Program, and focus on three issues: the role of perfectionism in the pre-treatment phase, the transformations perfectionism undergoes during treatment, and its role as a mediation factor between therapeutic alliance and outcome. The importance of forming a solid alliance with patients with high perfectionism is advocated, so as to boost interpersonal skills and thus achieve favorable outcomes, especially in shortterm psychodynamic treatment.

Werbart, Aldén, and Diedrichs (2017) have long been active in exploring the implications of Blatt's two configurations on the outcome of psychoanalytic treatment, contributing to the issue with a study that compares and contrasts a predominantly anaclitic group $(n=13)$ with an introjective one $(n=20)$. What makes this paper especially valuable is the particular attention given to post-treatment follow-up evaluations, usually rather difficult to undertake. Results showed that the anaclitic group displayed a better balance between relatedness and self-definition in the post-treatment phase, while this improvement was not significant in the introjective group. As other authors have correctly pointed out, further studies are needed to verify 
these findings, but nonetheless the diverse impact of the two configurations on psychoanalytic treatment was further substantiated, in keeping with literature findings.

In their first contribution, Dagnino and colleagues (de la Parra, Dagnino, Valdés, \& Krause, 2017) outline a theoretical and clinical model that sheds light on the potential relationship between depression and attachment, via the evaluation of the role of personality styles (dependent versus self-critical) and social support in this association. Here too, self-criticism appears to play a clearer role than dependency in mediating between attachment styles and depression. At the same time, social support seems to play a big part. These findings encourage us to think of depression as a disorder comprising diverse variables with manifold interconnections.

The second contribution by Dagnino and colleagues (Dagnino, Pérez, Gómez, Gloger, \& Krause, 2017) seeks to probe more thoroughly the self-criticism configuration, which is typical of the functioning of patients going through depressive experiences and which is deeply involved in the development of depression. Other key functions distinguishing this configuration - such as mentalization - are detailed and are contrasted with those associated with dependency. While self-critical functioning appears to be associated with vulnerabilities in regulating object relationships and attachments to internal objects, dependent functioning is linked to vulnerabilities in self-perception, self-regulation, and attachments to external objects. These findings are clearly not without significance for treatment methods and outcomes.

Clinical practice comes to the forefront in the contribution by Oasi and colleagues (2017). Here two patients with diverse depressive configurations are compared, after having been assessed prior to treatment and monitored regularly for the year-long course of the latter. In this approach, various instruments were employed with two key aims: to evaluate the possible link between personality and clinical syndrome and to evaluate how changes in this link affect defensive functioning and the psychotherapeutic relationship. A rich and intriguing picture emerges, in which Depressive Experiences Questionnaire (DEQ) measurements of depressive dimensions remain unchanged notwithstanding variations in other personality dimensions measured by Shedler-Westen Assessment Procedure (SWAP)-200.

On the topic of personality assessment and, more specifically, of how patients are depressed, the paper by Falgares et al. (2017) offers a contribution to the Italian validation of the DEQ. Indeed, to date, there is still no validated version of this self-report questionnaire in Italian. This study of the instrument involved three different universities, located in Southern, Central and Northern Italy. While results are promising, the authors also report some negative findings with regard to the dimension of dependency, an unexpected outcome in light of the numerous prior studies validating this construct.

All in all, this issue attests to the strength of Blatt's model, providing several instances of how the latter has managed to inform very diverse areas of research. We do not take Blatt's model as a form of reductionism, even though it posits two key dimensions in personality functioning, personality development, and psychopathology. In truth, it is to the intricacy of the interaction between relatedness and self-definition that we should look. Likewise, we should not overlook their meaningful coupling with other important constructs, such as attachment. It is our hope that this issue may constitute a spur for researchers to keep exploring Sidney Blatt's theories, as well as a basis for a wider dissemination of his ideas in Italy, where he is still somewhat unknown.

\section{References}

Auerbach, J. (2017). The contributions of Sidney J. Blatt: a personal and intellectual biography. Research in Psychotherapy: Psychopathology, Process and Outcome, 20(1), 3-11.

Dagnino, P., Pérez, J.C., Gómez, A., Gloger, S., \& Krause, M. (2017). Depression and attachment: how do personality styles and social support influence this relation? Research in Psychotherapy: Psychopathology, Process and Outcome, 20(1), 53-62.

de la Parra, G., Dagnino, P., Valdés, C., \& Krause, M. (2017). Beyond self-criticism and dependency: structural functioning of depressive patients and its treatment. Research in Psychotherapy: Psychopathology, Process and Outcome, 20(1), 43-52.

Falgares, G., De Santis, S., Gullo, S., Carrozzino, D, Marchetti, D., Verrocchio, M.C., ..., \& Oasi, O. (2017). The Italian version of the Depressive Experiences Questionnaire: psychometric properties and validation in students, community, and clinical groups. Research in Psychotherapy: Psychopathology, Process and Outcome, 20(1), 81-90.

Lingiardi, V., McWilliams, N., \& Muzi, L. (2017). The contribution of Sidney Blatt's two-polarities model to the psychodynamic diagnostic manual. Research in Psychotherapy: Psychopathology, Process and Outcome, 20(1), 12-18.

Miller, R., Hilsenroth, M., \& Hewitt, P. (2017). Perfectionism and therapeutic alliance: a review of the clinical research. Research in Psychotherapy: Psychopathology, Process and Outcome, 20(1), 19-29.

Oasi, O., Buonarrivo, L., Codazzi, A., Passalacqua, M., Risso Ricci, G.M., Straccamore, F., \& Bezzi, R. (2017). Assessing personality change with Blatt's anaclitic and introjective configurations and SWAP-200 profiles: two case studies in psychodynamic treatment. Research in Psychotherapy: Psychopathology, Process and Outcome, 20(1), 63-80.

Werbart, A., Aldén, S., \& Diedrichs, A. (2017). Changes in the anaclitic-introjective personality configurations following psychoanalytic psychotherapy with young adults. Research in Psychotherapy: Psychopathology, Process and Outcome, 20(1), 30-42. 